\title{
Assessment of a Multi-Agent RFID Platform for Industrial Logistic Operations
}

\author{
Zenon Chaczko ${ }^{1}$, Christopher $\mathrm{Chiu}^{2}$, Zhengyu $\mathrm{Yu}^{3}$ \\ Faculty of Engineering and Information Technology, University of Technology, Sydney \\ $\left\{{ }^{1}\right.$ zenon.chaczko, ${ }^{2}$ christopher.chiu, ${ }^{3}$ zhengyu. yu\}@uts.edu.au
}

\begin{abstract}
The paper examines the experimental implementation of multiagent, omni-dimensional RFID technology for logistical and industrial operations. The aim of the research is to demonstrate the processes taken to test RFID under real-world industrial scenarios using a multi-agent analytical approach. The results obtained demonstrate the level of accuracy for an omni-directional versus agent-based optimized analytical approach, that is essential in conveying the performance in industrial logistic setups. Further work into the positioning and localization of multidimensional RFID inventory scanning for commercial setups is considered for future investigation.
\end{abstract}

Keywords. Radio Frequency Identification (RFID), Supervisory Control and Data Acquisition (SCADA), Omni-directional Scanning and Tracking, Multi-dimensional Positioning Analysis, Agent-based Neural Networks, Logistics and Inventory Management

\section{Introduction}

Radio Frequency Identification (RFID) is a highly robust and available technology used in industrial and commercial applications, used for more than five decades since its introduction for military purposes [5]. Current RFID solutions are based on uni-directional scanning approaches, that is, where the geography of the readers is positioned in defined locations [2]. The purpose of this research is to investigate the practical applicability and performance of multi-dimensional scanning, combined with multi-dimensional analytics, to provide modeling of simulated inventory in space.

The practical applications of item tracking in commercial scenarios, including the monitoring of key inventory in industrial workflows, and predictive estimation of inventory processes for future forecasting of business operations [9]. The tracking of inventory orders is a key aspect in the modernization and automation of inventory management [4]. Real-time tracking does not only provide an exceptional edition to the user experience, it also aims to dramatically reduce the rate of stock shrinkage and loss occurring in current factory setups where readers are placed in fixed positions. 


\section{Conceptual Design}

\subsection{Planning and Deployment}

The RFID tracking of items is a critical element of the experimental setup, to determine the practical effectiveness of multi-dimensional tracking for further processing and modeling $[9,10]$. The requirements of the system were determined to satisfy the following criteria:

1. RFID Reader Detection of Individual Tag Positions: This requirement is important because it allows the system to determine whether a tag is located anywhere within the system. This way the system knows if an order has been completed and a tray has been removed; and

2. RFID Reader Calculated Location of Tag: This requirement complements the first requirement as it is also desirable to know the exact location of the tray in the system. This is also desirable to ensure visual identification of the experimental results.

\subsection{Laboratory Configuration}

The overall design of RFID system was designed and implemented around the entire experimental system. The arrangement of the RFID system was designed to provide relatively accurate and constant tracking of the fluctuating locations of the RFID tags. Nonetheless, the structure of the RFID system relied heavily on the tag being utilized.

Consequently, the structure of the RFID system had various modifications throughout the course of the experimental scenario, however, the structure and functions of the RFID reader and tags remained consistent throughout the design procedure. The complete setup as shown in Figure 1 on the facing page of the RFID implementation consisted of:

- RFID Reader (Impinj Xway Gateway GX31M1): Impinj omni-directional reader located 1.9 meters above the experimental set;

- RFID Tag (Xerafy UHF RFID Passive Tag): Iso 15693 compliant tag located underneath the tray, with all tags placed in the same location; and

- Software Implementation: Local terminal running C\# application built using the Microsoft Visual Studio Integrated Development Environment.

The measured setup was important to determine as this facilitated accurate results of the moving RFID tags, particularly if the tags were moved outside of the experimental environment. Thus, this allowed the determination of exact tag locations. Moreover, the design of the software was structured around the needs of the experimental requirements in the following criteria:

- Modification of output results into a format recognized by the experimental software system; 


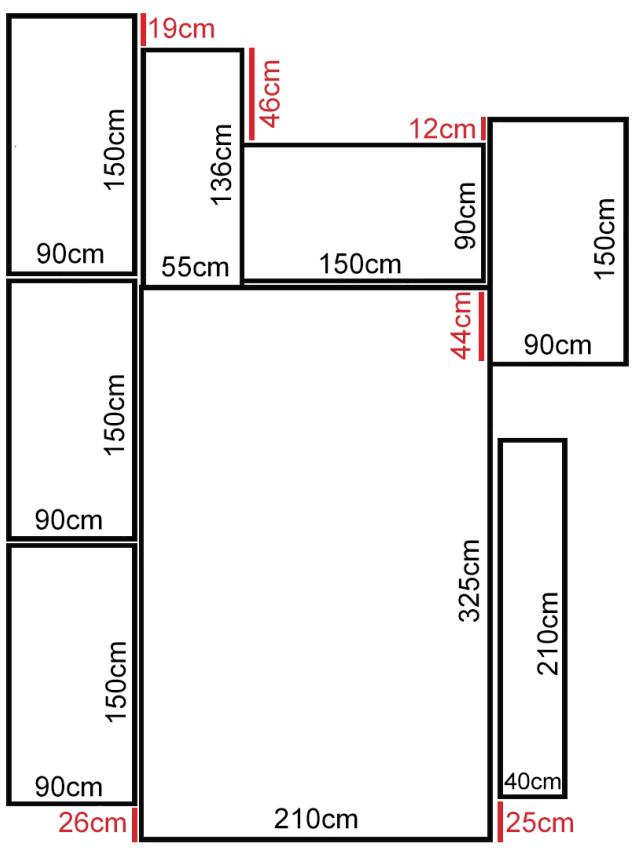

Fig. 1. Complete test factory setup measurements

- Manipulating results into a digital file stream, that were used to generate graphs that illustrated the location of the tag based on its accurate position on the factory setup;

- Offsetting results due to the RFID reader being in an offset location; and

- Incorporation of web-sockets into the software program to allow further integration with the SCADA and user interface.

Overall, the design, implementation and integration of RFID with the overall experimental system, is critical in ensuring the constant monitoring of the product order. As can be seen from Figure 2 on the next page, the location and orientation of the tag was critical, as this was replicated with all the other trays. This allowed for consistency and uniformity in results, especially due to the scattering of signals and the inaccuracy that came with the RFID reader.

\section{Implementation Planning}

\subsection{Hardware: RFID Transceiver}

The implementation of the Impinj RFID transceiver is mounted 1.9 meters above the experimental table as shown in Figure 3 on page 5. The mounting was made to be parallel to the physical environment, to ensure the reported locations of the tags are as close as approximately possible in the same $\mathrm{X}$ and $\mathrm{Y}$ axis as the 


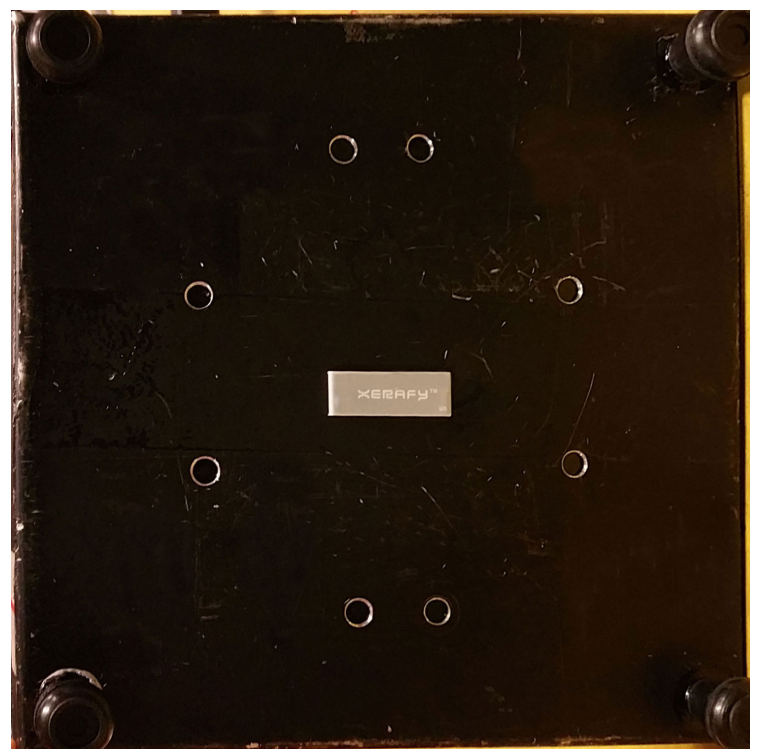

Fig. 2. Photograph of experimental tray setup with passive RfiD tag at center

table. This is to ensure the minimization of significant errors in the location of the RFID tags from the transceiver's perspective, with the radial zones of the tags relative to the transceiver shown in Figure 4 on the next page.

As observed from the transceiver setup, the alignment of the RFID reader must be consistent with the positioning of the experimental setup, in regards to the origin offsetting to obtain correct locations to match the anticipated results. Aligning the orientation of the RFID reader to meet the radial sectors predefined within the RFID reader was critical, to ensure it corresponded closely to the locations of the RFID tags in the laboratory setup. The matching of the $\mathrm{X}$ and $\mathrm{Y}$ axis between the reader output and the experimental system layout is achieved by mounting the RFID reader as parallel as possible with the table setup. This translated into a simple offset of the RFID reader output to integrate the final data analysis with the SCADA interface.

The physical alignment of the RFID transceiver respective to the lab setup is an important task to establish correctly, to ensure the theoretical system model faithfully reflects the mathematical model incorporated into the code of the experimental software system.

Technical Limitations The initial system setup has deficiencies that limit the accurate depiction of a typical inventory scenario, limiting the practicality of the experimental setup to be replicated in a real-world environment. These main limitations are identified along with the solutions in Table 1 on page 6 . 


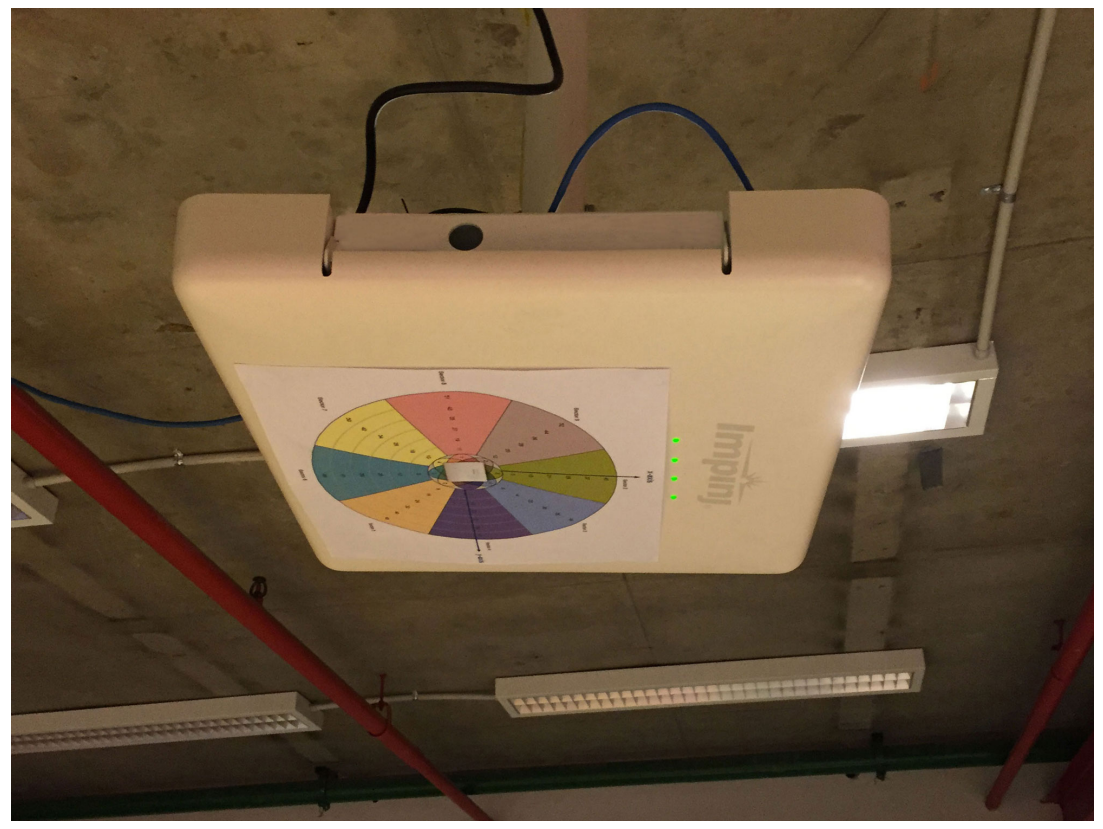

Fig. 3. Photograph of experimental RFID reader positioned above lab setup

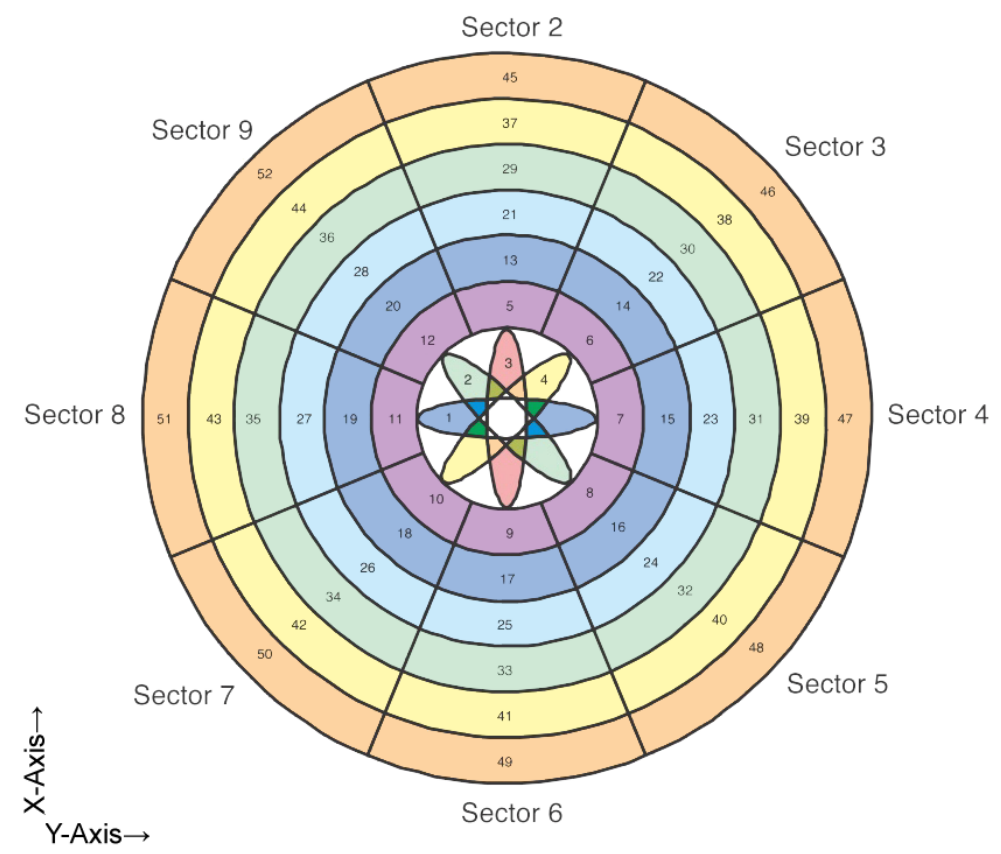

Fig. 4. Radial sector zones relative to Impinj RFID transceiver [10] 


\begin{tabular}{|c|c|}
\hline Identified Hardware Limitation & Mitigated Alternate Solution \\
\hline $\begin{array}{l}\text { - RFID reader improperly aligned, pro- } \\
\text { ducing inaccurate results that did not } \\
\text { match orientation. }\end{array}$ & $\begin{array}{l}\text { - Mounting the RFID reader as close as } \\
\text { parallel allowed the reader space to } \\
\text { move, which enabled it to be aligned. }\end{array}$ \\
\hline $\begin{array}{l}\text { - RFID tags are affected by multipath } \\
\text { distortion due to the signals reflecting } \\
\text { off the metallic objects. } \\
\text { - RFID tags are affected by scattering } \\
\text { due to the RF signals impinging onto } \\
\text { objects whose dimensions were smaller } \\
\text { than the electromagnetic waves, thus } \\
\text { signal strength was diminished. }\end{array}$ & $\begin{array}{l}\text { - Re-positioning the arms of the robots } \\
\text { mitigated this problem. } \\
\text { - Clearing the area of small metallic } \\
\text { and/or non-penetrable objects miti- } \\
\text { gated this problem. } \\
\text { - During operation of the experiment } \\
\text { this cannot be mitigated or avoided. }\end{array}$ \\
\hline $\begin{array}{l}\text { - The read quality of certain tags was } \\
\text { sub-optimal and was not suitable for } \\
\text { the experimental system. }\end{array}$ & $\begin{array}{l}\text { - A range of experiments was undertaken } \\
\text { on numerous tags to distinguish which } \\
\text { tag would be sufficient for the setup. }\end{array}$ \\
\hline
\end{tabular}

Table 1. Hardware Limitations and Mitigated Solutions

\subsection{Software: Design and Implementation}

The software system identifies the manufacturer name for each tag for tracking purposes, to easily distinguish which tag was returning which result, especially as a typical commercial environment would have numerous tags being read near the RFID reader. Thus, it was important for the code to identify the correct tag description, as this was vital in the comparison of tracking approaches.

The system requires an optimal level of logging verbosity, outputting the necessarycoordinate data on the console window whilst in operation $[2,8]$. The RFID monitoring software implementation should not impact on the performance of the Impinj RFIDreader that would affect the results, ensuring a manageable and user friendly experience $[1,7]$. The source code should not contain redun-dant functionality that would impact on tracking performance, and allow for debugging and future code portability. 


\section{Conclusion}

The multi-agent RFID localization platform, through its design and implementation, evaluates the effectiveness of a hybridized approach using hardware and software-based localization of RFID assets. The demonstration of the localization platform, employing an open distributed architecture with web-sockets, shows the potential feasibility of further amalgamation of SCADA infrastructure such that logistic operations can assess and analyze their inventory management strategies in an automated fashion.

A functional prototype of a logistical environment is designed to show the potential real-world concerns of omni-directional RFID localization. The experimental system captures localization data from the RFID reader, with the ability for end-users to examine in real-time which localization methodologies is most suitable for their operational logistic procedures. Employing localization modeling enables the user to determine the feasibility of using omni-directional RFID technologies in warehousing facilities. Further work with the post-processed data can be examined using specialized data mining algorithms to plan positional strategies and predict inventory transaction trends.

\section{References}

[1] Barbacci, M. et al, 1996. Principles for Evaluating the Quality Attributes of a Software Architecture, Carnegie Mellon University, Software Engineering Institute, pp33-34

[2] Booch, G., 1991. Object-Oriented Analysis and Design with Applications, AddisonWesley, p 51

[3] Chaczko, Z. et al, 2006. 7/24 Software Development in Virtual Student Exchange Groups: Redefining the Week, Iтнет '06, Sydney, Australia, July 2006

[4] Chaczko, Z., Davis, J.D., Mahadevan, V., 2004. New Perspectives on Teaching and Learning Software Systems Development in Large Groups, 5th ITHEт Conference 2004, p278

[5] Chaczko, Z., Davis, J.D., Scott, C., 2004. New Perspectives on Teaching and Learning Software Development in Large Group Telecollaboration, IADIS International Conference 2004 Madrid, Spain, 6-9 October 2004

[6] Chaczko, Z., Marcel, M., Lim, L., 2005. Middleware Model for Telecollaborative Systems in Education, Iтнет '05, Dominican Republic, July 2005

[7] Jacobson, I., 1998. Object-Oriented Software Engineering: A Use Case Driven Approach: Revised Edition, Addison-Wesley, pp57-58

[8] Kruchten, P., 1996. A Rational Development Process, Software Technology Support Centre Crosstalk Forum, July 1996

[9] O'Brien, L. et al, 1991. Retailing: Shopping, Society, Space, David Fulton Publishers, London, pp71-75

[10] Yang, J. et al, 2003. Smart Market Assistants and Recreation Trolley, Beijing Institute of Technology, China, Beijing 